\title{
Distributed Algorithms for Coloring and Domination in Wireless Ad Hoc Networks
}

\author{
Srinivasan Parthasarathy ${ }^{1}$ and Rajiv Gandhi ${ }^{2}$ \\ 1 Department of Computer Science, University of Maryland, College Park, MD 20742. Research supported by NSF Award \\ CCR-0208005. E-mail: sri@cs.umd.edu. \\ 2 Department of Computer Science, Rutgers University, Camden, NJ 08102. E-mail: rajivg@camden.rutgers .edu. Part of \\ this work was done when the author was a student at the University of Maryland and was supported by NSF Award \\ CCR-9820965. Research also supported by Rutgers University's Research Council grant.
}

\begin{abstract}
We present fast distributed algorithms for coloring and (connected) dominating set construction in wireless ad hoc networks. We present our algorithms in the context of Unit Disk Graphs which are known to realistically model wireless networks. Our distributed algorithms take into account the loss of messages due to contention from simultaneous interfering transmissions in the wireless medium.

We present randomized distributed algorithms for (conflict-free) Distance-2 coloring, dominating set construction, and connected dominating set construction in Unit Disk Graphs. The coloring algorithm has a time complexity of $O\left(\Delta \log ^{2} n\right)$ and is guaranteed to use at most $O(1)$ times the number of colors required by the optimal algorithm. We present two distributed algorithms for constructing the (connected) dominating set; the former runs in time $O\left(\Delta \log ^{2} n\right)$ and the latter runs in time $O\left(\log ^{2} n\right)$. The two algorithms differ in the amount of local topology information available to the network nodes.

Our algorithms are geared at constructing Well Connected Dominating Sets (WCDS) which have certain powerful and useful structural properties such as low size, low stretch and low degree. In this work, we also explore the rich connections between WCDS and routing in ad hoc networks. Specifically, we combine the properties of WCDS with other ideas to obtain the following interesting applications:

- An online distributed algorithm for collision-free, low latency, low redundancy and high throughput broadcasting.

- Distributed capacity preserving backbones for unicast routing and scheduling.
\end{abstract}

\section{Introduction}

Wireless ad hoc networks are composed of a set of mobile nodes which communicate with one another over a shared wireless channel. Unlike wired networks, nodes in an ad hoc network do not rely on a pre-existing communication infrastructure. Instead, they communicate either directly with each other or with the help of intermediate nodes in the network. The distributed, wireless and self-configuring nature of ad hoc networks render them useful for several applications such as mobile battlefields, disaster relief, sensing and monitoring. However, the lack of a fixed communication infrastructure introduces several challenging and interesting research issues in the design of communication protocols for these networks. Any communication protocol for ad hoc networks should also contend with the issue of interference in the wireless medium. When two or more nodes transmit a message to a common neighbor at the same time, the common node will not receive any of these messages. In such a case, we say that a collision has occurred at the common node.

Coloring and connected domination are two fundamental primitives with several applications in the wireless context. In wireless networks, we seek a conflict-free coloring of the nodes such that two nodes which belong to the same color class may transmit simultaneously without resulting in collisions. Clearly, such a coloring has natural applications to collision-free wireless scheduling. In order to overcome the lack of a fixed routing infrastructure, several researchers have also proposed construction of a virtual backbone in ad hoc networks. A virtual backbone typically consists of a small subset of nodes in the network which gather and maintain information such as local topology and traffic conditions. This information can be made use of by higher level protocols for providing efficient communication services. Connected Dominating Sets (CDS) are the earliest structures proposed as candidates for virtual backbones in ad hoc networks [11, 10,29].

Both coloring and (connected) dominating set construction are classical problems which have received tremendous attention in the literature. In general, all existing distributed algorithms for these problems can be classified into 
two categories. The first category of algorithms are fast sub-linear time algorithms which do not consider message losses due to collisions. Further, these all algorithms model the network as an arbitrary undirected graph; both these assumptions render them unsuitable for wireless ad hoc networks. The second category of algorithms are (slower) linear time algorithms. These algorithms can be implemented such that only a single node in the network transmits at any time and hence no collisions occur during the course of the algorithm. A linear time algorithm does not exploit the massive parallelism available in the ad hoc network and is unsuitable for dynamic network conditions displayed by ad hoc networks.

In this work, we focus on developing fast distributed algorithms for coloring and (connected) dominating set construction in wireless ad hoc networks. Specifically, we view the following as the main contributions of this work.

\subsection{Our Contributions}

- Incorporating Wireless interference: We present distributed algorithms for conflict-free coloring, dominating set construction and connected dominating sets in the context of wireless networks. While several distributed algorithms exist for coloring and domination in arbitrary graphs, we use Unit Disk Graphs which realistically model wireless networks. Further, our algorithms handle wireless interference; we take into account the loss of messages at a node due to collisions from simultaneous neighboring transmissions. We are not aware of any work which study these problems under message losses due to wireless collision.

- Distributed Coloring We present a distributed conflict-free (D2) coloring of nodes in the network. This primitive arises naturally in many applications such as broadcast scheduling and channel assignment in wireless networks. In general, the colors could represent time slots or frequencies assigned to the nodes. Minimizing the number of colors used in the coloring is very desirable for these applications, but is known to be NP-hard [28]. Our algorithm runs in time $O\left(\Delta \log ^{2} n\right)$, where $\Delta$ is the maximum degree and $n$ is the number of network nodes and uses $O(\Delta)$ colors for the D2-coloring; this is at most $\mathrm{O}(1)$ times the number of colors used by an optimal algorithm.

- Distributed (Connected) Dominating Set: We present distributed algorithms for dominating set and connected dominating set construction where require knowledge of only local topology and global network parameters such as size and the maximum degree. We present two algorithms: a D2-coloring based algorithm and a broadcast based algorithm which utilizes the work of Gandhi et al. [13]. The coloring based algorithm requires each node to know the maximum degree $\Delta$ and the total number of network nodes $n$ and runs in time $O\left(\Delta \log ^{2} n\right)$. The broadcast based algorithm requires each node to know their three-hop topology and runs in time $O\left(\log ^{2} n\right)$. All these algorithms incorporate message losses due to collisions from interfering transmissions.

- Wireless Routing Applications: The distributed CDS algorithms presented in this paper are geared at constructing CDSs with certain powerful structural properties such as low size, low stretch and low degree (henceforth, we refer to such a CDS as a Well Connected Dominating Sets (WCDS)). The work by Alzoubi [2] deals with a linear-time distributed construction of WCDS in ad hoc networks. In this paper, we also explore the rich connections between WCDS and routing in wireless networks. Specifically, we combine the structural properties of WCDS with other ideas to obtain the following interesting applications:

- An online distributed algorithm for collision-free, low latency, low redundancy and high throughput broadcasting.

- Distributed capacity preserving backbones for unicast routing and scheduling.

We note that our algorithms and analysis only require that nodes know a good estimate of the values of the network parameters $n$ and $\Delta$ instead of their exact values. Such estimates are easy to obtain in many practical scenarios. For instance, consider the scenario where $n$ nodes with unit transmission radii are randomly placed in a square grid of area $n$. In this case, the maximum degree $\Delta=\Theta\left(\frac{\log n}{\log \log n}\right)$ with high probability.

The remainder of this paper is organized as follows: in section 2, we introduce the basic models and definitions which will be used in the rest of the paper. We survey related work in section 3. In section 4, we present our D2coloring algorithm. In section 5 and 6 , we present our two distributed algorithms for dominating set construction and CDS construction respectively. Sections 7 and 8 deal with the applications of WCDS to broadcast and unicast routing and scheduling respectively. Due to lack of space, we omit the proofs of all the claims presented in this paper. All the proofs appear in the full version of this work. ${ }^{3}$

\footnotetext{
${ }^{3}$ Available at http://www.cs.umd.edu/ sri/distcoldom.ps
} 


\section{Background}

\subsection{Network and Interference Model}

We model the network connectivity using a unit disk graph (UDG) $G=(V, E)$ : the nodes in $V$ are embedded in the plane. Each node has a maximum transmission range and an edge $(u, v) \in E$ if $u$ and $v$ are within the maximum transmission range of each other. We assume that the maximum transmission range is the same for all nodes in the network (and hence w.l.o.g., equal to one unit). Time is discrete and synchronous across the network; units of time are also referred to as time slots. Since the medium of transmission is wireless, whenever a node transmits a message, all its neighbors hear the message. If two or more neighbors of a node $w$ transmit at the same time, $w$ will be unable to receive any of those messages. In this case we also say that $w$ experiences collision. In any time slot, a node can either receive a message, experience collision, or transmit a message but cannot do more than one of these. We work with the above interference model for ease of exposition and analysis. However, all the results presented in this paper easily extend to the so called protocol model [16] of interference also.

\subsection{Definitions}

We now describe the definitions and notations used in the rest of the paper. All the definitions below are with respect to the undirected graph $G=(V, E)$.

Connected Dominating Set (CDS): A set $W \subseteq V$ is a dominating set if every node $u \in V$ is either in $W$ or is adjacent to some node in $W$. If the induced subgraph of the nodes in $W$ is connected, then $W$ is a connected dominating set (CDS). A Minimum Connected Dominating Set (MCDS) is a CDS with the minimum number of nodes.

Maximal Independent Set (MIS): A set $M \subseteq V$ is an independent set if no two nodes in $M$ are adjacent to each other. $M$ is also a Maximal Independent Set (MIS) if there exists no set $M^{\prime} \supseteq M$ such that $M^{\prime}$ is an independent set. Note that, in an undirected graph, every MIS is a dominating set.

Well Connected Dominating Set (WCDS): A CDS $W$ is a WCDS if it satisfies the following properties:

(P1) Low Size: Let $O P T$ be an MCDS for $G$. Then, $|W| \leq k_{1}|O P T|$, where $k_{1}$ is a constant.

(P2) Low Degree: Let $G^{\prime}=\left(W, E^{\prime}\right)$ be the graph induced by the nodes in $W$. For all $u \in W$, let $d^{\prime}(u)$ denote the degree of $u$ in $G^{\prime}$. Then, $\forall u \in W, d^{\prime}(u) \leq k_{2}$, where $k_{2}$ is a constant.

(P3) Low Stretch: Let $D(p, q)$ denote the length of the shortest path between $p$ and $q$ in $G$. Let $D_{W}(p, q)$ denote the length of the shortest path between $p$ and $q$ such that all the intermediate nodes in the path belong to $W$. Let $s_{W} \doteq \max _{\{p, q\} \in V} \frac{D_{W}(p, q)}{D(p, q)}$. Then, $s_{W} \leq k_{3}$, where $k_{3}$ is a constant.

Distance-k Neighborhood (Dk-neighborhood): For any node $u$, the Dk-neighborhood of $u$ is the set of all other nodes which are within $k$ hops away from $u$.

Distance-2 Vertex Coloring (D2-coloring): D2-coloring is an assignment of colors to the vertices of the graph such that every vertex has a color and two vertices which are D2-neighbors of each other are not assigned the same color. Vertices which are assigned the same color belong to the same color class. This definition is motivated by the fact that nodes belonging to the same color class can transmit messages simultaneously without any collisions.

\section{Related Work}

Coloring, dominating set construction and connected domination are classical problems which have been extensively studied in the literature. However, we are not aware of any distributed algorithms for these problems which incorporate the geometry and transmission characteristics of wireless networks. To the best of our knowledge, we are the first to study these problems for realistic multi-hop wireless network models (Unit Disk Graphs) and incorporate loss of messages due to collisions from interfering transmissions.

Distance-2 coloring is a basic primitive used in wireless networks and is well studied in the context of broadcast scheduling and channel assignment $[28,27,25]$. In [28], it was shown that even in the case of UDGs, it is NP-hard to minimize the number of colors used in the D2-coloring. However, for many restricted graph classes such as UDGs, 
several centralized approximation algorithms exist which use within $\mathrm{O}(1)$ times the number of colors used by an optimal D2-coloring $[28,19,26]$. In this paper, we present the first distributed algorithm for D2-vertex coloring in UDGs with $\mathrm{O}\left(\Delta \log ^{2} n\right)$ running time and which uses at most $\mathrm{O}(1)$ times the number of colors used by an optimal D2-coloring. We note that several related problems have received attention in the case of single-hop wireless networks which can be modeled as a clique (see [18] for instance).

CDS was first proposed as a candidate for virtual backbones in [11,10,29]. Guha and Khuller [15] studied the Minimum Connected Dominating Set (MCDS) problem and showed that computing an MCDS is NP-hard in arbitrary undirected graphs. They also presented centralized approximation algorithms which are guaranteed to produce a CDS of size $\mathrm{O}(\log n)$ times that of an MCDS. Dubashi et al.[12] present a fast distributed CDS algorithm for undirected graphs with a running time of $\mathrm{O}\left(\log ^{3} n\right)$. In addition, the solution produced by their algorithm has size $\mathrm{O}(\log \Delta)$ times MCDS and stretch $\mathrm{O}(\log n)$. However, this algorithm does not take into account the loss of messages due to collisions in wireless network. Although this algorithm is very attractive for general networks, it is not directly applicable to wireless ad hoc networks. The algorithms in $[30,33]$ construct a CDS of size $\mathrm{O}(n)$ times MCDS and stretch $\mathrm{O}(1)$ in arbitrary undirected graphs. The time complexity of the algorithm in [33] is $\Theta\left(\Delta^{3}\right)$. However, these algorithms also do not consider message losses due to collisions in their model.

It was shown in [9] that computing an MCDS is NP-hard even for UDGs. Cheng et al.[8] propose a centralized polynomial time approximation scheme (PTAS) for approximating MCDS in UDGs. Several distributed approximation algorithms exist for computing MCDS in UDGs $[32,24,3,4,6]$. These algorithms produce a solution whose size is within $\mathrm{O}(1)$ times that of an MCDS. The time and message complexity of these algorithms are $\mathrm{O}(n)$ and $\mathrm{O}(n \log n)$ respectively. All these algorithms have a stretch of $O(n)$ [2]. Alzoubi et al.[5] proposed a distributed CDS algorithm for UDGs which has $\mathrm{O}(n)$ time and message complexity and which results in a CDS of size O(1) times MCDS. Recently, Alzoubi [2] showed that this CDS also has $\mathrm{O}(1)$ stretch. We improve upon the time complexity of all the above algorithms by proposing the first sub-linear time distributed algorithms for ad hoc networks which constructs a WCDS of size O(1) times MCDS and O(1) stretch. In particular, we note that in comparison with [2], we achieve a drastic decrease in the time complexity (from $\mathrm{O}(n)$ to $\mathrm{O}\left(\log ^{2} n\right)$ at the expense of a slight increase in the message complexity (from $O(n)$ to $O(n \log n)$ ). While the distributed algorithm presented in [2] holds for both synchronous and asynchronous models of communication, we restrict our focus only to the synchronous communication model and leverage in the design of our distributed algorithms.

During CDS construction, it is often desirable to treat domination and connectivity as orthogonal requirements (as we do in our work). The best known results for dominating set in UDGs are a constant factor approximation algorithm by Alzoubi et al. [1] and by Gao et al. [14]. The former is a linear time distributed algorithm; the latter also shows how to continuously maintain the dominating set thus computing under node mobility, however, it does not account for wireless interference during the dominating set construction and maintenance. For general graphs, the best known distributed algorithms for dominating sets are the ones by Kuhn et al. [20] and Jia et al. [17]. They present distributed logarithmic approximation algorithms for dominating sets in general graphs which run in constant and polylogarithmic time respectively. Again, neither of these two algorithms are meant to handle wireless interference.

Network-wide broadcasting is an important application for CDS in ad hoc networks. Several CDS based broadcasting algorithms exist where only the nodes in the CDS are involved in retransmitting the messages. Gandhi et al.[13] proposed a collision-free distributed broadcast scheme for UDGs, which broadcasts a set of offline messages. In this scheme, both the number of retransmissions and the broadcast latency is within $\mathrm{O}(1)$ times their optimal values. We improve upon this result in this paper by proposing a WCDS based online collision-free broadcast algorithm, which guarantees low latency, low number of retransmissions and high throughput, all within $\mathrm{O}(1)$ times their optimal values.

Luby $[22,23]$ proposed randomized distributed algorithms for vertex coloring and MIS construction in arbitrary undirected graphs. We adapt these algorithms for D2-coloring and MIS construction in UDGs. Luby's algorithms were originally meant for a system of parallel processors. Our adaptations which are meant for wireless ad hoc networks are complicated by the fact that messages can be lost due to collisions. Topkis [31] analyzed the time-complexity of flooding for all-to-all broadcasting in a wired network. We use some of the proof techniques from [31] in our analysis WCDS based broadcasting in Section 6.

Kumar et al.[21] propose algorithms for unicast packet scheduling under the D2-edge interference model. We utilize one of their results on end-to-end unicast scheduling to obtain our results in section 7. Our results in Section 
7 are motivated by the work of Chen et al. [7], which proposes a distributed algorithm for constructing a forwarding backbone, which preserves the routing capacity of the underlying network.

\section{Distributed D2-Coloring}

In this section, we present our distributed D2-coloring algorithm for unit disk graphs. Our algorithm is modeled after Luby's distributed graph coloring algorithm [23]. The key technical difficulty in our algorithm as opposed to Luby's algorithm, lies in the the fact that simultaneous transmissions from neighboring nodes could result in collisions and hence loss of messages at a particular node. We handle this by probabilistic retransmission of the messages, and ensure that all messages are eventually received by their intended recepients with high probability. Further, while Luby's distributed coloring algorithm was a D1-coloring of arbitrary graphs, our algorithm is intended for D2-coloring of unit disk graphs. This allows us to exploit the geometric properties of UDGs to D2-color it using $O(\Delta)$ colors; this yields a $O(1)$ approximation for the number of colors.

Our algorithm is parametrized by three positive integers: $c, t$, and $r$ (to be specified later). Each node $u$ has a list of colors $L(u)$ which is initialized to $\{1,2, \ldots c\}$. Time is divided into frames of length c time slots. As in Luby's algorithm [23], our algorithm also proceeds in a synchronous round by round fashion. Typically, each round involves the following steps. Some of the yet-uncolored nodes choose a tentative colors for themselves. Some of these nodes will be successful, since none of their D2-neighbors would have chosen the same tentative color as themselves. In this case, the tentative color becomes the permanent color for these nodes. The unsuccessful nodes update their color list by removing the set of colors chosen by their successful D2-neighbors in this round and continue their attempts to color themselves in the future rounds. The coloring algorithm terminates after $t$ rounds. We now present the details of a specific round.

Each round consists of four phases: TRIAL, TRIAL-REPORT, SUCCESS and SUCCESS-REPORT. The details of these phases are given below.

TRIAL: Only the yet-uncolored nodes participate in this phase. This phase consists of a single frame. At the beginning of this phase, each yet-uncolored node $u$ wakes up or goes to sleep with probability $1 / 2$ respectively. If $u$ is awake, it chooses a tentative color color $(u)$ uniformly at random from $L(u)$. Note that $L(u)$ is the list of colors available for node $u$ in the current round and this list may change in the future rounds. Node $u$ then transmits a TRIAL message $\{I D(u)$, color $(u)\}$ at the time slot corresponding to color $(u)$ in this frame: for e.g., if $u$ is awake and if color $(u)=5, u$ transmits the message $\{I D(u), 5\}$ at the fifth time slot of this frame. In general, the TRIAL message (and other types of messages below) may not reach all the neighbors of $u$ due to collisions.

TRIAL-REPORT: This phase consists of $r$ frames. At the beginning of this phase, every node $u$ in the network prepares a TRIAL-REPORT message. This message is the concatenation of all the TRIAL messages received by $u$ in this round. During every frame of this phase, $u$ chooses a time slot independently at random within the frame, and broadcasts the TRIAL-REPORT message during this time.

SUCCESS: This phase consists of a single frame. At the beginning of this phase, every node $u$ which is awake, determines if the tentative color it chose during the TRIAL phase is a safe color or not. Intuitively, color $(u)$ is safe if no node in its D2-neighborhood chose the same color as $u$. In our algorithm, $u$ deems color $(u)$ to be safe if the following conditions hold:

1. $u$ received a TRIAL-REPORT message from each of its neighbors.

2. Each TRIAL-REPORT message received by $u$ contained the TRIAL message sent by $u$.

If the above conditions are met, color $(u)$ becomes the permanent color for $u$. In this case, $u$ creates a SUCCESS message $\{I D(u)$, color $(u)\}$ and broadcasts it to all its neighbors. This transmission is done at the time slot corresponding to color $(u)$ within this frame. In future rounds, $u$ does not participate in the TRIAL and SUCCESS phases since it successfully colored itself in this round.

SUCCESS-REPORT: This phase is similar to the TRIAL-REPORT phase. The SUCCESS-REPORT message for every node $u$ in the network is a concatenation of SUCCESS messages which were received by $u$ in this round. This phase also consists of $r$ frames. During every frame of this phase, $u$ chooses a time slot independently at random within the frame and broadcasts its SUCCESS-REPORT message during this slot. Crucially, at the end of 
this phase, any yet-uncolored node $v$ removes from its list $L(v)$, any color found in the SUCCESS or SUCCESSREPORT messages received by $v$ in this round. This ensures that, in the future rounds, $v$ does not choose the colors of its successful D2-neighbors.

This completes the description of a single round of the algorithm; the algorithm consists of $t$ such rounds. We show that for an appropriate choice of parameters, our algorithm yields a $O(1)$-approximate D2-coloring for UDGs with high probability in $O\left(\Delta \log ^{2} n\right)$ time. Specifically, let the parameters have the following values: $c=k_{1} \Delta, t=k_{2} \log n$, and $r=k_{3} \log n$, where $k_{1}, k_{2}$ and $k_{3}$ are constants. The following theorem holds.

Theorem 1. The distributed D2-coloring algorithm computes a valid D2-coloring using $O(\Delta)$ colors in $O\left(\Delta \log ^{2} n\right)$ running time w.h.p. The number of colors used is at most $O(1)$ times the optimal coloring. All messages in the algorithm require at most $O(\Delta \log n)$ bits. The total number of messages transmitted by the algorithm is at most $O\left(n \log ^{2} n\right)$.

\section{Distributed Dominating Set Construction}

In this section, we present our distributed dominating set algorithms for unit disk graphs. We note that any Maximal Independent Set (MIS) is also a dominating set in an undirected graph. Further, in the case of UDGs, it is well known that the number of nodes in any Maximal Independent Set (MIS) is at most five times the number of nodes in the minimum dominating set. Hence, a distributed MIS algorithm also yields a 5-approximate dominating set in UDGs. Henceforth, we focus on distributed MIS construction in UDGs.

\subsection{D2-coloring based MIS Algorithm}

We now present a simple D2-coloring based distributed MIS algorithm. Observe that if we have a D2-coloring of the nodes using $c$ colors, we can build an MIS iteratively in $c$ time slots as follows: during slot $i$, all nodes belonging to color class $i$ attempt to join the MIS. A node joins the MIS if and only if none of its neighbors are currently part of the MIS. After joining the MIS, the node broadcasts a message to its neighbors indicating that it joined the MIS. Nodes transmitting during the same time slot belong to the same color class and hence do not share a common neighbor. For the same reason, none of the messages are lost due to collisions. Clearly, this stage requires exactly $c$ time steps. Since the distributed D2-coloring algorithm of Section 4 colors the UDG using $O(\Delta)$ colors w.h.p. in $O\left(\Delta \log ^{2} n\right)$ time, we also have a distributed MIS algorithm which terminates correctly w.h.p. in $O\left(\Delta \log ^{2} n\right)$ time.

Theorem 2. The D2-coloring based distributed algorithm constructs an MIS in $O\left(\Delta \log ^{2} n\right)$ time w.h.p and the total number of messages transmitted during the algorithm is $O\left(n \log ^{2} n\right)$.

\subsection{Broadcast based MIS construction}

We now present our broadcast based distributed MIS algorithm, which makes use of knowledge of the Distance-2 topology, and constructs an MIS in $O\left(\log ^{2} n\right)$ time. Specifically, we assume that each node knows its D2-neighborhood and the edges between these nodes. As in Luby's distributed MIS algorithm [22], our algorithm also proceeds in a synchronous round by round fashion. The MIS is initially empty. Typically, some nodes are successful at the end of each round. A node is deemed successful if either the node joins the MIS or one of its neighbors joins the MIS. Successful nodes do not participate in the future rounds (except for forwarding messages), while remaining nodes continue their attempts to be successful in the future rounds. The MIS construction terminates after $t$ such rounds.

During the algorithm, each node $u$ maintains a status variable which is defined as follows: status $(u)=$ in if $u$ has joined the MIS; $\operatorname{status}(u)=$ out if any neighbor of $u$ has joined the MIS; status $(u)=$ unsure otherwise. All nodes are initially unsure and become in or out of MIS during the course of the algorithm. Let $V_{i}$ be the set of nodes whose status is unsure at the end of round $i-1$. For any node $u \in V_{i}$, let $N_{i}(u)=N(u) \cap V_{i}$. Let $M I S_{i}$ be the set of nodes which join MIS in round $i$.

There are four phases in each round of the algorithm: TRIAL, CANDIDATE-REPORT, JOIN, and PREPARE. We now present the details of these phases for a particular round $i$. 
TRIAL: In this phase, each unsure node decides if it is a candidate for $M I S_{i}$. Specifically, each unsure node $u$ chooses itself to be a candidate for joining $M I S_{i}$, with probability $\frac{1}{2\left(\left|N_{i}(u)\right|+1\right)}$. Node $u$ will not be a candidate in this round with the complement probability. This phase does not required any message transmissions.

CANDIDATE-REPORT: This phase ensures that each node knows if there is a neighbor who is a candidate. This step consists of $p$ time frames, each frame consisting of two slots. During every frame of this phase, each candidate node chooses one of the two slots independently at random and broadcasts a CANDIDATE message. Any node which receives a CANDIDATE message or experiences collision during this phase, knows that there is a neighboring candidate; otherwise it assumes that there is no neighboring candidate.

JOIN: This phase requires a single time slot. In this phase, some unsure nodes become either in or out. How should a candidate decide if it should join $M I S_{i}$ (become in)? A candidate joins $M I S_{i}$ if none of its neighbors are candidates for $M I S_{i}$, i.e., if it did not receive a CANDIDATE message during the previous phase. All nodes who joined $M I S_{i}$ transmit a JOIN message. unsure nodes which receive a JOIN message or experience collision, change their status to out. Other unsure nodes do not change their status.

PREPARE: Each unsure node $u$ computes $N_{i+1}(u)$ at the end of this phase. This phase consists of $p$ time frames. Each frame is further subdivided into $\alpha$ sub-frames of length $c$. During every frame of this phase, each node in $M I S_{i}$, chooses independently at random, one of the $\alpha$ sub-frames. During this sub-frame, it broadcasts a PREPARE message using the algorithm in [13] to its D2-neighbors. The length of the sub-frame, $c$ is the number of time steps required by [13] to transmit a message from a node to its D2-neighbors. The PREPARE message broadcast by a node simply consists of its ID. By the end of this phase, every unsure node knows all the nodes in its D2-neighborhood which joined $M I S_{i}$. Hence, it can easily compute $N_{i+1}(u)$.

The algorithm terminates after $t$ such rounds. The theorem below claims that for an appropriate choice of parameters, the algorithm yields an MIS with high probability in time $O\left(\log ^{2} n\right)$. The analysis of this theorem involves a tricky charging argument which heavily relies on the geometry of UDGs.

Theorem 3. The broadcast based distributed algorithm computes an MIS with high probability in $O\left(\log ^{2} n\right)$ time. Each message is at most $O(\log n)$ bits in length and the expected number of messages transmitted is $O(n \log n)$.

\section{Distributed Connected Domination}

In this section, we present our distributed connected dominating set algorithms for UDGs. Alzoubi [2] presented a centralized algorithm for constructing a CDS with a stretch of $O(1)$, size which is at most $O(1)$ times that of the minimum CDS, and has $O(1)$ degree. Henceforth, we will call a CDS with these properties as Well Connected Dominating Set (or WCDS). Alzoubi also presented a distributed implementation of his centralized algorithm which runs in linear time. The basic idea behind the centralized algorithm is as follows: we first compute an MIS by iteratively choosing vertices which are currently not in MIS and which do not currently have a neighbor in MIS. Since the input graph is an undirected graph, any maximal independent set is also a dominating set. Connectivity is handled as an orthogonal component as follows: every MIS node $u$ is connected to every other MIS node $v$ in its D3-neighborhood, using a shortest path between $u$ and $v$. Nodes in the shortest paths along with the nodes in MIS constitute the CDS $W$. We now present two distributed implementations of this approach.

\subsection{D2-Coloring based CDS Algorithm}

Our first distributed algorithm is based on the D2-coloring algorithm and runs in $O\left(\Delta \log ^{2} n\right)$. Briefly this algorithm has three stages. The first stage involves computing a distributed D2-coloring using $O(\Delta)$ colors and in $O\left(\Delta \log ^{2} n\right)$ time. The second stage involves computing an MIS in $O(\Delta)$ steps as described in Section 4. The third stage involves connecting each MIS node to its all its D3-neighbors. We now describe the details involved in the third stage.

This stage requires six phases. Each phase is one frame long and a single frame is of length $c=O(\Delta)$, where $c$ is the number of colors in the D2-coloring. Nodes transmit only during the time slot corresponding to their D2-color. During the first phase, all MIS nodes transmit a PHASE-1 message. This message just consists of the node's ID. In the second phase, any node $u$ which received a PHASE- 1 message, transmits a PHASE-2 message. This message is a 
concatenation of $I D(u)$ and all the PHASE-1 messages received by $u$. In the third phase, any node $u$ which received a PHASE-2 message, transmits a PHASE-3 message. This message is a concatenation of $I D(u)$ and all the PHASE-2 messages received by $u$.

By the end of the third phase, every MIS node $u$ knows every other MIS node $v$ in its D3-neighborhood. Node $u$ also knows all paths of length at most three between itself and $v$. Node $u$ constructs a PHASE-4 message as follows: for every other MIS node $v$ such that $v$ is in its D3-neighborhood and $I D(v)>I D(u), u$ chooses a path of length at most three hops between itself and $v$. It adds this information to its PHASE-4 message. All MIS nodes transmit a PHASE-4 message during the fourth phase. Every node $u$ which received a PHASE-4 message transmits a PHASE-5 message. This message is a concatenation of all the PHASE- 4 messages received by $u$. Finally every node $u$ which received a PHASE-5 message transmits a PHASE-6 message. This message is a concatenation of all the PHASE-5 messages received by $u$. By the end of this stage, any MIS node $u$ knows the path between itself and any other MIS node $v$ which in its D3-neighborhood. In addition, any node $w$ which is not part of the MIS, knows if it is part of the final WCDS or not.

This completes the description of the algorithm. It is easy to see that this algorithm yields a WCDS w.h.p (since, the correctness of the WCDS depends only upon the correctness of the D2-coloring algorithm). Further, its running time is clearly dominated by the time required to compute the D2-coloring which is $O\left(\Delta \log ^{2} n\right)$.

Theorem 4. The D2-coloring based CDS algorithm computes a WCDS in time $O\left(\Delta \log ^{2} n\right)$ w.h.p.

\subsection{Broadcast based CDS Algorithm}

We now describe our broadcast based distributed CDS algorithm which runs in time $O\left(\log ^{2} n\right)$ time. In this algorithm, we assume that each node knows its D3-topology. This algorithm involves two stages. The first stage is basically a distributed MIS construction as described in Section 5.2 which runs in $O\left(\log ^{2} n\right)$ time. The second stage involves connecting each MIS node to its three-hop MIS neighbors. This stage consists of two phases: HELLO and CONNECT. We now present the details of these phases.

HELLO: The objective of this phase is for each MIS node to announce itself to other MIS nodes in its D3neighborhood. This phase consists of $p$ frames, where each frame is subdivided into $\alpha^{\prime}$ sub-frames of length $c^{\prime}$ (these constants will be specified later). During every frame of this phase, each node $u \in M I S$ node selects independently at random, one of the $\alpha^{\prime}$ sub-frames. During this sub-frame, $u$ broadcasts a HELLO message using the algorithm in [13] to its D3-neighborhood (this is where the knowledge D3-topology is required). By the end of this phase, each MIS node knows any other MIS node in its D3-neighborhood.

CONNECT: This phase is similar to the HELLO phase. The only difference is in the contents of the CONNECT message. Each node $u \in M I S$ prepares its CONNECT message as follows. For every node $v \in M I S$ such that $v$ is in its D3-neighborhood and $I D(v)>I D(u)$, the CONNECT message of $u$ contains the tuple $\{I D(v), u \sim v\}$. $u \sim v$ is the shortest path between $u$ and $v$. As mentioned earlier, CONNECT messages are broadcast in the same way as the HELLO messages. Intermediate nodes, which are not part of the MIS, might join the WCDS, since they might be a part of the shortest path between two MIS nodes. This completes the description of our distributed broadcast based WCDS algorithm. Let $\alpha^{\prime}$ be the maximum number of MIS nodes in the D3-neighborhood of a node. Let $c^{\prime}$ be the maximum time required by the broadcast algorithm in [13] to broadcast a message from a node to its D3-neighborhood. The following theorem holds.

Theorem 5. The broadcast based CDS algorithm computes a WCDS in time $O\left(\log ^{2} n\right)$ w.h.p. Further, each message is of size $O(\log n)$ and the total number of messages transmitted in expectation is $O(n \log n)$.

The time and message complexity of this algorithm is essentially dominated by the MIS construction. Further, we note that the proof of correctness of the HELLO and CONNECT phases are identical to the PREPARE phase of the broadcast based MIS construction (except that these messages are broadcast to the D3-neighborhood unlike the PREPARE message which goes to the D2-neighborhood). 


\section{$7 \quad$ Network-wide Broadcasting}

We now present a simple, online, collision-free, distributed algorithm for broadcasting messages across the network using a WCDS $W$. For ease of analysis, we assume that messages are generated only by nodes in $W$. Our algorithm requires that nodes in $W$ have a valid D2-coloring. Specifically, let $G^{\prime}$ be the induced subgraph of nodes in $W$. The maximum degree of any node in $G^{\prime}$ is a constant (follows from (P2), section 2.2). Hence, it is possible to D2-color all nodes in $W$ using only $k$ colors, where $k$ is a constant. This pre-processing can be done using the D2-coloring stage of the algorithm in section 4 . We let $0,1, \ldots, k-1$ denote the set of colors and color $(u)$ denote the color of node $u$.

Our broadcast scheme requires that each message has a unique sequence number associated with it. This assumption is needed in order to enforce a total ordering of broadcast messages generated in the network. This can be easily ensured in practice by labeling each message $\mu$ with a triplet $\langle T(\mu), S(\mu), L S N(\mu)\rangle$. Here, $T(\mu)$ is the time at which message $\mu$ was generated. $S(\mu)$ is the source of message $\mu . L S N(\mu)$ is a locally generated sequence number for message $\mu$ at its source $S(\mu)$. It is easy to verify that this labeling is sufficient to enforce a total ordering of the messages.

Let time be divided into frames of length $k$. Let $Q(\mu)$ denote the frame during which message $\mu$ was generated, i.e., $Q(\mu) k \leq T(\mu)<(Q(\mu)+1) k$. Let $0,1, \ldots$ be the (totally ordered) set of broadcast messages generated in the network. Since the network is multi-hop, intermediate nodes need to assist in the broadcast operation by retransmitting the messages. In our scheme, only nodes in $W$ retransmit messages. Every node $u \in W$ retransmits every message exactly once. Let $R(u, q)$ denote the set of messages which have been received by node $u$ by the end of frame $q$. Node $S(\mu)$ is deemed to have received message $\mu$ during frame $Q(\mu)$. Let $X(u, q)$ denote the set of messages which have been retransmitted by node $u$ by the end of frame $q$. The following simple rule specifies the behavior of any node $u \in W$ during a particular frame $q$.

- Let $P=R(u, q-1) \backslash X(u, q-1)$. If $P$ is empty, then $u$ does not retransmit any message in frame $q$. Otherwise, let $\lambda=\min (P)$ be the least numbered message in $P$. Node $u$ transmits message $\lambda$ in the time slot corresponding to $\operatorname{color}(u)$ in frame $q$.

This simple scheme guarantees that all nodes in the network receive all messages collision-free. In addition, this scheme optimizes the latency, the number of retransmissions, and the throughput of the broadcast to within a constant factor of their respective optimal values. We analyze the behavior of our broadcast algorithm under the following packet injection model.

Imagine an "adversary" who generates broadcast messages in the network. The long term rate at which the adversary can generate messages is at most a constant, although he can generate messages in bursts. Specifically, let $G^{\prime}$ denote the induced subgraph of $W$, and let $R^{\prime}$ denote the diameter of $G^{\prime}$. Recall that $k$ is the number of colors required for D2-coloring the WCDS. During any continuous window of length $2 k R^{\prime}$ time slots, our adversary is allowed to generate at most $R^{\prime}$ messages. Thus the long term term message generation rate of our adversary is at most $\frac{1}{2 k}$, which is a constant. Note that no broadcast protocol can support more than a unit long term rate, at most one message can be received by a receiver at every time slot. We now state the following theorem under this adversarial behavior.

Theorem 6. The latency experienced by any message $\mu$ is $O(R)$. This is at most $O(1)$ the latency experienced by the message in an optimal broadcast algorithm since any algorithm incurs a latency of $R$. All messages are received collision-free by all nodes in the network. In addition, the number of retransmissions for any message is at most $O(1)$ times the optimal number of retransmissions required to broadcast the message.

\section{Unicast Routing}

In this section, we show that a WCDS is an efficient backbone for unicast routing in ad hoc networks. We derive our results in this section under the Distance-2 edge interference model (D2-model) [25, 26, 21]. 


\subsection{Distance-2 Edge Interference Model (D2-model)}

The D2-model is motivated by wireless communication protocols such at 802.11 , where a single transmission along an edge involves the transmission of the data from one end point and the transmission of an acknowledgment from the other end point. Thus both the end points behave as senders and receivers (of data and ack) during a single transmission along an edge. This is in contrast with the scenarios encountered so far where in each transmission, only one end point of the edge behaves as a sender and the other behaves as a receiver.

Specifically, let $(u, v)$ and $(p, q)$ be edges in the network. We say that $(p, q)$ interferes with $(u, v)$ if $p$ or $q$ is a neighbor of $u$ or $v$. A transmission along the edge $(u, v)$ is considered collision-free if there is no other transmission along any edge $(p, q)$ which interferes with $(u, v)$.

\subsection{Efficient Backbones for Unicast Routing}

Two critical components for any routing algorithm are its path selection and scheduling strategies. The path selection strategy determines the path along which each packet traverses in the network. The scheduling strategy determines the time at which a packet is transmitted along each edge on its path. The scheduling component also ensures that the packets are transmitted collision-free (one may think of these components as the network and the MAC layer protocols respectively). Together, they uniquely determine the latency experienced by every packet in the network.

Our goal here is not to present algorithms for path selection or scheduling. Instead, we show that any routing algorithm could be modified to operate over a WCDS. Crucially, the modified routing algorithm will use only the nodes in WCDS as intermediate nodes in the paths, without incurring significant loss in the quality of the paths and schedules when compared with the original algorithm. We formalize this intuition below.

Let $\mathcal{P}=\left\{p_{1}, \ldots p_{n}\right\}$ be a set of paths such that the maximum length of any path is $d$. We will refer to the elements of $\mathcal{P}$ as both paths and packets interchangeably. For any disk $z$, let $n(z)$ denote the number of edges in all the paths in $\mathcal{P}$ with an end point inside $z$. Let $Z$ be the set of all disks on the plane with radius $1 / 2$. Let $c=\max _{z \mid z \in Z} n(z)$ : i.e., $c$ is the maximum number of edges in $\mathcal{P}$ which have an end point inside any fixed disk of radius $1 / 2$. We call $d$ and $c$, the dilation and congestion of $\mathcal{P}$ respectively. A schedule $S$ for $\mathcal{P}$ specifies the time at which every packet is transmitted collision-free along each edge in its path. The length of the schedule $|S|$ is the maximum latency of any packet in this schedule, i.e., the maximum time at which any packet traverses any edge. Observe that, under the D2-model, both $c$ and $d$ (and hence $\frac{c+d}{2}$ ) are lower bounds on the length of any schedule for $\mathcal{P}$. We now state the following surprising claim from [21].

Claim. Let $O P T$ be an optimal collision-free schedule for $\mathcal{P}$ under the D2-model. Let $|O P T|$ denote the length of $O P T$ (which is the maximum latency experienced by a packet in $O P T$ ). Then, $|O P T|=\Theta(c+d)$.

This result implies that there always exists a schedule in which all packets reach their destinations collision-free such that the maximum latency experienced by any packet is at most $\Theta(c+d)$ (and not $\Theta(c d)$ ).

We now construct a new set of paths $\mathcal{P}^{\prime}$ from $\mathcal{P}$ as follows. Consider any path $p=\left(u=u_{0} \rightarrow u_{1} \rightarrow u_{2} \ldots u_{l}=v\right)$ in $\mathcal{P}$. W.L.O.G., let $u$ and $v$ not belong to $W$. For any node $x$, let $\operatorname{dom}(x)$ denote the dominator of $x$ in $W$. For each $p \in P$, we create $p^{\prime} \in \mathcal{P}^{\prime}$ such that $p^{\prime}=\left(u=u_{0} \rightarrow \operatorname{dom}\left(u_{0}\right) \leadsto \operatorname{dom}\left(u_{1}\right) \leadsto \operatorname{dom}\left(u_{2}\right) \ldots \operatorname{dom}\left(u_{l}\right) \rightarrow u_{l}=v\right)$. Here $\operatorname{dom}(a) \leadsto \operatorname{dom}(b)$ is the path of length at most 3 from $\operatorname{dom}(a)$ to $\operatorname{dom}(b)$ in $W$. Let $d$ and $d^{\prime}$ be the dilation of $\mathcal{P}$ and $\mathcal{P}^{\prime}$ respectively. Let $c$ and $c^{\prime}$ be the congestion of $\mathcal{P}$ and $\mathcal{P}^{\prime}$ respectively. We now state the following theorem without proof (which we omit due to lack of space).

Theorem 7. $c^{\prime}+d^{\prime}=\Theta(c+d)$.

This theorem states that the new set of paths $\mathcal{P}^{\prime}$ through the unicast routing backbone, does not experience significantly more congestion or dilation than the original set of paths $\mathcal{P}$. Thus, by claim 8.2 , there exists a schedule $S^{\prime}$ in which every packet reaches from its source to its destination collision-free with a maximum latency of $\Theta\left(c^{\prime}+d^{\prime}\right)=$ $\Theta(c+d)$.

Intuitively, this result implies that any a schedule for a set of packets in the original network can be converted into a new schedule for this set of packets on top of the WCDS. The new schedule will use only the nodes in the 
WCDS for routing. However, the latency experienced of the new schedule is at most $O(1)$ times the latency of the original schedule. In this sense, the WCDS preserves the capacity of the underlying network.

Acknowledgments. We would like to thank V.S. Anil Kumar, Madhav Marathe and Aravind Srinivasan for several useful discussions.

\section{References}

1. K. Alzoubi, P.-J. Wan, and O. Frieder. Maximal independent set, weakly connected dominating set, and induced spanners for mobile ad hoc networks. International Journal of foundations of Computer Science (IJFCS), Special Issue on Wireless, 2003.

2. K. M. Alzoubi. Connected dominating set and its induced position-less sparse spanner for mobile ad hoc networks. In Proceedings of the Eighth IEEE Symposium on Computers and Communications, June 2003.

3. K. M. Alzoubi, P.-J. Wan, and O. Frieder. Distributed heuristics for connected dominating sets in wireless ad hoc networks. IEEE ComSoc/KICS Journal on Communication Networks, 4:22-29, 2002.

4. K. M. Alzoubi, P.-J. Wan, and O. Frieder. Message efficient construction of nontrivial connected dominating sets in wireless ad hoc networks. To appear in Special Issue of ACM Journal of Monet, 2002.

5. K. M. Alzoubi, P.-J. Wan, and O. Frieder. Message-optimal connected-dominating-set construction for routing in mobile ad hoc networks. In Proceedings of the Third ACM International Symposium on Mobile Ad Hoc Networking and Computing, June 2002.

6. K. M. Alzoubi, P.-J. Wan, and O. Frieder. New distributed algorithm for connected dominating set in wireless ad hoc networks. IEEE HICSS35, 2002.

7. Benjie Chen, Kyle Jamieson, Hari Balakrishnan, and Robert Morris. Span: An energy-efficient coordination algorithm for topology maintenance in ad hoc wireless networks. In Proceedings of the 7th annual international conference on Mobile computing and networking, pages 85-96. ACM Press, 2001.

8. X. Cheng, X. Huang, D. Li, and D.-Z. Du. Polynomial-time approximation scheme for minimum connected dominating set in ad hoc wireless networks. Technical report.

9. B. Clark, C. Colbourn, and D. Johnson. Unit disk graphs. Discrete Mathematics, 86:165-177, 1990.

10. B. Das, R. Sivakumar, , and V. Bharghavan. Routing in ad-hoc networks using a virtual backbone. In 6th International Conference on Computer Communications and Networks (IC3N '97), pages 1-20, September 1997.

11. Bevan Das and Vaduvur Bharghavan. Routing in ad-hoc networks using minimum connected dominating sets. In $I C C$ (1), pages 376-380, 1997.

12. Devdatt Dubhashi, Alessandro Mei, Alessandro Panconesi, Jaikumar Radhakrishnan, and Arvind Srinivasan. Fast distributed algorithms for (weakly) connected dominating sets and linear-size skeletons. In Proceedings of the fourteenth annual ACM-SIAM symposium on Discrete algorithms, pages 717-724. Society for Industrial and Applied Mathematics, 2003.

13. Rajiv Gandhi, Srinivasan Parthasarathy, and Arunesh Mishra. Minimizing broadcast latency and redundancy in ad hoc networks. In Proceedings of the fourth ACM international symposium on Mobile ad hoc networking and computing, pages 222-232. ACM Press, 2003.

14. Jie Gao, Leonidas Guibas, John Hershberger, Li Zhang, and An Zhu. Discrete mobile centers. In Proceedings of the seventeenth annual symposium on Computational geometry, pages 188-196. ACM Press, 2001.

15. Sudipto Guha and Samir Khuller. Approximation algorithms for connected dominating sets. Algorithmica, pages 374-387, 1998.

16. Piyush Gupta and P. R. Kumar. The capacity of wireless networks. IEEE Transactions on Information Theory, 46(2):388404, March 2000.

17. Lujun Jia, Rajmohan Rajaraman, and Torsten Suel. An efficient distributed algorithm for constructing small dominating sets. Distrib. Comput., 15(4):193-205, 2002.

18. Tomasz Jurdzinski and Grzegorz Stachowiak. Probabilistic algorithms for the wakeup problem in single-hop radio networks. In Proceedings of the thirteenth Annual International Symposium on Algorithms and Computation, 2002.

19. Sven O. Krumke, Madhav V. Marathe, and S. S. Ravi. Models and approximation algorithms for channel assignment in radio networks. Wireless Networks, 7(6):575-584, 2001.

20. Fabian Kuhn and Roger Wattenhofer. Constant-time distributed dominating set approximation. In Proceedings of the twenty-second annual symposium on Principles of distributed computing, pages 25-32. ACM Press, 2003.

21. V. S. Anil Kumar, Madhav V. Marathe, Srinivasan Parthasarathy, and Aravind Srinivasan. End-to-End Packet Scheduling in Ad Hoc Networks. To appear in ACM SODA 2004.

22. Michael Luby. A simple parallel algorithm for the maximal independent set problem. SIAM Journal on Computing, 15(4):1036-1053, November 1986.

23. Michael Luby. Removing randomness in parallel computation without a processor penalty. Journal of Computer and System Sciences, 47(2):250-286, 1993. 
24. Madhav V. Marathe, Heinz Breu, Harry B. Hunt III, S. S. Ravi, and Daniel J. Rosenkrantz. Simple heuristics for unit disk graphs. Networks, 25:59-68, 1995.

25. S. Ramanathan. A unified framework and algorithm for channel assignment in wireless networks. Wireless Networks, 5(2):81-94, 1999.

26. Subramanian Ramanathan and Errol L. Lloyd. Scheduling algorithms for multihop radio networks. IEEE/ACM Transactions on Networking (TON), 1(2):166-177, 1993.

27. Arunabha Sen and Mark L. Huson. A new model for scheduling packet radio networks. Wireless Networks, 3(1):71-82, 1997.

28. Arunabha Sen and Ewa Melesinska. On approximation algorithms for radio network scheduling. In Proceedings of the 35th Allerton Conference on Communication, Control and Computing, pages 573-582, 1997.

29. R. Sivakumar, B. Das, and V. Bharghavan. Spine routing in ad hoc networks. ACM/Baltzer Cluster Computing Journal (special issue on Mobile Computing), 1998.

30. I. Stojmenovic, M. Seddigh, and J. Zunic. Dominating sets and neighbor elimination based broadcasting algorithms in wireless networks. In Proceedings of IEEE Hawaii International Conference on System Sciences, January 2001.

31. Donald M. Topkis. All-to-all broadcast by flooding in communications networks. IEEE Transactions on Computeres, 38(9):1330-1333, 1989.

32. P.-J. Wan, K. Alzoubi, and O. Frieder. Distributed construction of connnected dominating set in wireless ad hoc networks. In IEEE INFOCOM, 2002.

33. J. Wu and H. L. Li. On calculating connected dominating set for efficient routing in ad hoc wireless networks. In Proceedings of the Third ACM International Workshop on Discrete Algorithms and Methods for Mobile Computing and Communications, pages 7-14, 1999. 